\title{
Analysis and Design of Position and Velocity Estimation Scheme for PM servo motor drive with Binary Hall Sensors
}

\author{
Qinan Ni \\ School of Electrical Engingeering and \\ Automation \\ Harbin Institute of Technology \\ Harbin, China \\ niqn_hit@163.com \\ Pericle Zanchetta \\ Department of Electrical and Electronic \\ Engineering \\ University of Nottingham \\ Nottingham, UK \\ Pericle.Zanchetta@nottingham.ac.uk
}

\author{
Ming Yang \\ School of Electrical Engingeering and \\ Automation \\ Harbin Institute of Technology \\ Harbin, China \\ yangming@hit.edu.cn \\ Xiaosheng Liu \\ School of Electrical Engingeering and \\ Automation \\ Harbin Institute of Technology \\ Harbin, China \\ liuxsh@hit.edu.cn
}

\author{
Shafiq Ahamed Odhano \\ Department of Electrical and Electronic \\ Engineering \\ University of Nottingham \\ Nottingham, UK \\ Shafiq.Odhano@nottingham.ac.uk \\ Dianguo Xu \\ School of Electrical Engingeering and \\ Automation \\ Harbin Institute of Technology \\ Harbin, China \\ xudiang@hit.edu.cn
}

\begin{abstract}
-with the increasing demand of low-cost, highefficiency, high performance for AC motor drive system, the permanent-magnet synchronous motor (PMSM) with binary Hall sensors begins to be adopted in many fields. Compared with sensorless control, the usage of binary Hall sensors is a guarantee for the drive to achieve moderate control performance, and it is in smaller volume and more costeffective compared with other types of position sensors. In this paper, a solution is provided to realize fully-closed loop control with low-resolution position sensors, by treating the position and speed estimators as separate systems. Results reveal that the model-based methods can take advantage of model information and model-free methods can smoothly process the quantized Hall position signal. Extensive experiment results are provided demonstrating the position control performance and basic servo performance for a PMSM drive using 3 bitper-pole-pair sensing system.
\end{abstract}

Keywords-Observer, polynomial fitting, binary Hall sensors, servo application

\section{INTRODUCTION}

The surface-mounted permanent magnet synchronous motor (SMPMSM), with advantages such as reliable operation, high efficiency and small volume, has gradually become the main trend of AC servo field. AC servo motor drives based on field-oriented control (FOC) have been widely applied in position control systems, whose performance is principally dependent on the accuracy of rotor angle and speed information. Generally, cost and reliability are the primary concerns in many servo fields, such as machine tools, robot arms, electric vehicle and home appliance applications, where the servo motor is operated with huge impact or vibration forces or it is driven in hightemperature and high-humidity environment conditions.

In order to avoid encoder troubles and to improve the servo system reliability, various sensorless techniques, or position sensorless techniques in other words, have been developed as an alternative measure of shaft-mounted sensors over the last two decades or so. However, estimation and control of the absolute (mechanical) rotor position has been rarely considered and it is indispensably required particularly in motion and position controls for servo drive

This Paper is Funded by Project of Key Scientific and Technological Achievements Commercialization of Hu Nan Province Strategic Emerging Industries (NO. 2016GK4049) applications [1]. In addition, it is well known that most of the sensorless methods are still not mature to realize the aim of low cost while guarantee its performance within entire speed and torque range for some mission-critical drives[2-5].

At the same time, as a practical compromise, the sensor board fixed to the stator including inexpensive binary Hall sensors is often employed since it requires little cost and volume compared with shaft-mounted sensors and provides discrete absolute rotor position information. As reported in [6], two Hall sensors produce position information with electrically $\pm 45^{\circ}$ resolution are the worst resolution for detecting the rotating direction of the motor. Incidentally, it is not easy to conduct the servo control at such a low resolution. The layout with three Hall sensors can provide a $\pm 30^{\circ}$ resolution and possess an inherent double redundancy to faults [7-8]. From these rough position measurements, moderate position control performance can be costeffectively achieved by estimating accurate speed and high resolution position through signal processing or error correction techniques [9-11].

In recent years, a lot of investigations on PMSM drives using binary Hall sensors have been done and many speed/position estimation approaches ranging from modelfree and model-based have been extensively studied [12-16]. Most of the model-based methods exploit observer, or Kalman filter (KF) techniques. These methods can take advantages of the information of plant models and feature high-accuracy estimation and good dynamic characteristic in speed estimation. However, it should notice that, the position error from the quantization always exists in the sampled position signals, and this error can be regarded as a kind of measurement noise. So in this noisy environment, bumps may exist in speed estimation. Furthermore, the rotor position is usually obtained by numerical integration method from the estimated speed. Hence, the estimated speed error can affect the position estimation directly, so it becomes an actual limitation factor of the overall system performance in cascaded-loop structure based servo motor drive. In addition, for practical application, the observer gains were tuned simply by considering the relation of pole locations in s- and z- planes. However, stability analysis for observer needs to be further and comprehensively studied in Hall sensor based drives where the sampling frequency of position changes 
with respect to the motor velocity. Moreover, the Kalman filter based methods usually require the knowledge of noise variance and assume that the quantization error is Gaussian noise, which is hard to be satisfied in low resolution sensor system. On the other hand, model-free methods usually exploit signal processing techniques, such as filtering techniques, curve fitting methods, or numerical integration. These methods calculate the estimated position by reconstruct Hall position information without using motor parameter and the speed can be easily obtained by numerical differentiation methods from the calculated position information. Hence, the problem of time delay in estimated speed is unavoidable, the speed control loop may become slower and inefficient, and sometimes even unstable in speed dynamics.

Finally, it is worth noticing that position/speed estimators can be treated as separated systems, since the model-based methods can take advantage of model information and model-free methods can smoothly process the quantized Hall position signal. This paper discusses a new method that combines them together, which consider both position control and speed servo control performances. In proposed algorithm, the position signal is reconstructed by a polynomial fitting approach based on a series of past position and time data, and the speed feedback is estimated by a multi-sampling rate observer. Besides, to reduce the algorithm computational load and improve the fitting accuracy, simple solutions are also considered in this paper.

The outline of this paper is as follows. In Section II, the performance limitations of two conventional methods are analyzed from perspectives of time delay and estimation errors. In Section III, the proposed scheme is introduced in detail, followed by tuning and estimation performance analysis. The experimental results verify the effectiveness of the proposed scheme in Section IV. Section V concludes the whole paper.

\section{Performance Limitation Analysis of CONVENTIONAL METHODS}

It is necessary that the resolution should be enhanced for achieving high-performance vector control for a sinusoidal, surface-mounted PM machine drive that uses low resolution position sensors. However, it is not easy to estimate the exact speed and rotor position in the low-cost mass products. Two conventional methods, i.e. average speed method and Luenberger observer method, are analysed in the following subparagraphs. The analysis shows that the two methods may introduce time delay and errors in the estimated position and speed, and this can influence both the dynamic and steady performances and also stability of PMSM servo system to some extent.

\section{A. Average speed method}

The average speed with three Hall sensors can be calculated by the time-based strategy as shown by following expression:

$$
\omega_{\text {avg }}\left(t_{k}\right)=\frac{\theta\left(t_{k}\right)-\theta\left(t_{k-1}\right)}{\Delta t}
$$

where $\Delta t$ is the time interval of the previous Hall sector, $\theta\left(t_{k}\right)$ is the quantized position measured from the Hall

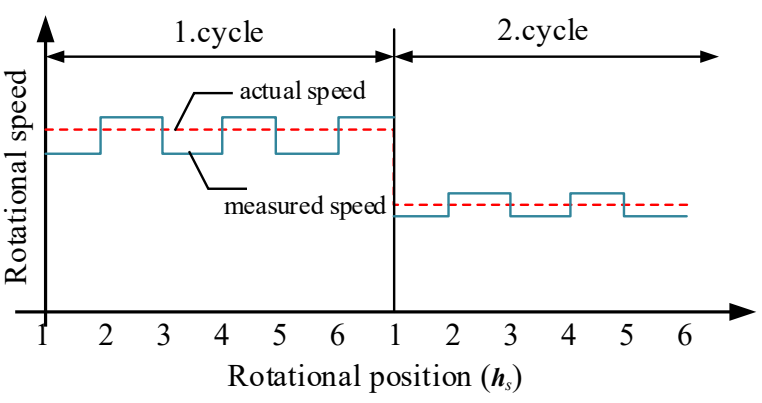

Fig. 1. Actual rotational speed and calculated average rotational speed

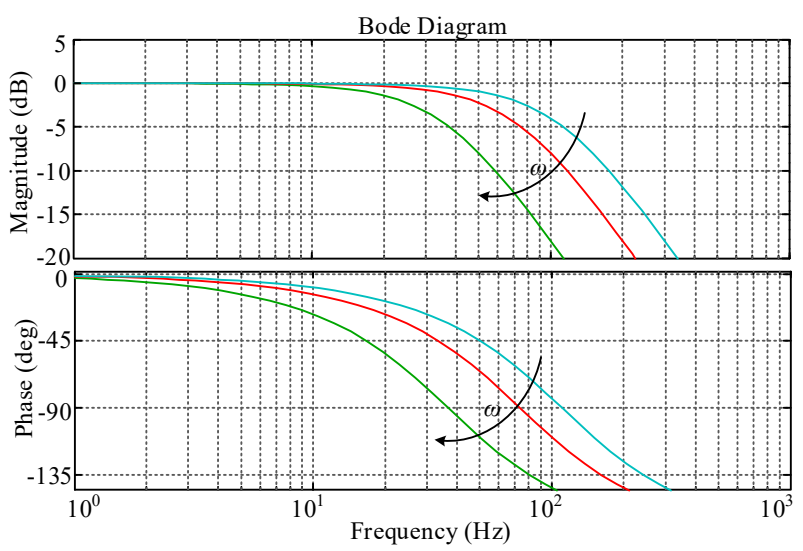

Fig. 2. Bode diagram of average speed method with MA filter

sensors, $\omega_{\text {avg }}$ is the calculated electrical rotor speed. Then, the rotor position can be obtained by numerical integration:

$$
\hat{\theta}_{h}\left(t_{k}+n \cdot T_{s}\right)=\theta\left(t_{k}\right)+n \cdot \omega_{\text {avg }}\left(t_{k}\right) \cdot T_{s}
$$

where $\hat{\theta}_{h}\left(t_{k}+n \cdot T_{s}\right)$ is the calculated rotor position in electrical angle at a sampling point $n, T_{\mathrm{s}}$ is the sampling frequency of current loop.

Applying Laplace transform, (1) becomes

$$
\hat{\Omega}_{\text {avg }}(s)=\frac{1-e^{-s \Delta t}}{s \Delta t} \Omega(s)=V(s) \Omega(s)
$$

where

$$
V(s)=\frac{1-e^{-s \Delta t}}{s \Delta t}
$$

However, even the machine rotates at constant speed, due to the inaccuracy in the detected position, $\Delta t$ may exit $\pm T_{\mathrm{s}}$ sampling error and the calculated speed by (1) has an error and the resultant interpolated position by (3) has the discontinuity wherever the Hall signal occurs, as shown in Fig. 1. The simplest solution is to low pass filter the estimated speed and to compute the average of the last $N$ samples, giving rise to the medium average (MA) filter as follows:

$$
F(s)=\frac{1}{N} \sum_{i=0}^{N-1} e^{-s i \Delta t}
$$

Then, the transfer function between the estimated and actual velocity can be described as: 


$$
\begin{aligned}
\frac{\hat{\Omega}_{m}(s)}{\Omega_{m}(s)} & =H(s)=V(s) F(s) \approx \frac{1-e^{-s \Delta t}}{s \Delta t} \frac{1}{N} \sum_{i=0}^{N-1} e^{-s i \Delta t} \\
& =\frac{1}{s N \Delta t}\left(\sum_{i=0}^{N-1} e^{-s \Delta t}-e^{-s \Delta t} \sum_{i=0}^{N-1} e^{-s \Delta t}\right) \\
& =\frac{1}{s N \Delta t} \sum_{i=0}^{N-1}\left(e^{-s \Delta t}-e^{-s(i+1) \Delta t}\right) \\
& =\frac{1}{s N \Delta t}(\underbrace{1-e^{-s \Delta t}}_{i=0}+\underbrace{e^{-s \Delta t}-e^{-s 2 \Delta t}}_{i=1}+\cdots+\underbrace{e^{-s(N-1) \Delta t}-e^{-s N \Delta t}}_{i=N-1}) \\
& =\frac{1-e^{-s N \Delta t}}{s N \Delta t}
\end{aligned}
$$

It's magnitude and phase depend on the number of samples in the MA filter, $N$, and on the sampling of Hall signals, $\Delta t$, as follows:

$$
\begin{gathered}
|T(j \omega)|=\frac{|\sin (\omega N \Delta t)|}{\omega N \Delta t} \\
\angle T(j \omega)=-\omega N \Delta t
\end{gathered}
$$

Thus, the average speed method with MA filter acts as a low pass filter whose effects on the velocity (see Fig. 2), in terms of amplitude and phase distortion, increase as $\mathrm{N}$ increases and real speed decreases.

Due to the delay of the estimated velocity in the lowspeed region, the speed control loop becomes slower and inefficient, and sometimes even unstable.

\section{B. Luenberger observer method}

In order to extract potentially "zero-lag" estimates of position and velocity, a number of methods have been proposed. Among these, the method utilizing a Luenberger observer illustrated in Fig. 3 has superior dynamic characteristics and high accuracy in estimation, such observer has intrinsic zero-lag tracking capability.

Assuming the friction coefficient of the system is 0 , thus the state equation of the observer can be expressed as

$$
\begin{aligned}
\dot{\hat{x}} & =A \hat{x}+B u+L(y-\hat{y}) \\
\frac{d}{d t}\left[\begin{array}{c}
\hat{\theta} \\
\hat{\omega} \\
\hat{T}_{d}
\end{array}\right] & =\left[\begin{array}{ccc}
0 & 1 & 0 \\
0 & 0 & -1 / \hat{J} \\
0 & 0 & 0
\end{array}\right]\left[\begin{array}{c}
\hat{\theta} \\
\hat{\omega} \\
\hat{T}_{d}
\end{array}\right]+\left[\begin{array}{c}
0 \\
1 / \hat{J} \\
0
\end{array}\right] T_{e}+\left[\begin{array}{l}
l_{1} \\
l_{2} \\
l_{3}
\end{array}\right]\left(\theta_{h}-\hat{\theta}\right)
\end{aligned}
$$

where, $\theta_{h}, T_{\mathrm{d}}, T_{e}$, and $J$ represent quantized Hall position signal, load torque, electromagnetic torque, and rotational inertia, respectively. $L$ is the observer gain. $y$ represents the output variables, and its value is $\hat{y}=C \hat{x}=\left[\begin{array}{lll}1 & 0 & 0\end{array}\right] \hat{x}$.

The transfer function for the speed, $\hat{\omega}$, can be deduced as follows:

$$
\hat{\omega}=\frac{\hat{J} l_{1} s^{3}+\hat{J} l_{2} s^{2}-l_{3} s}{\hat{J}^{3}+\hat{J} l_{1} s^{2}+\hat{J} l_{2} s-l_{3}} \theta_{h}+\frac{s^{2}}{\hat{J}^{3}+\hat{J} l_{1} s^{2}+\hat{J} l_{2} s-l_{3}} T_{e}
$$

In (8), the quantized Hall position and the torque reference can be described as

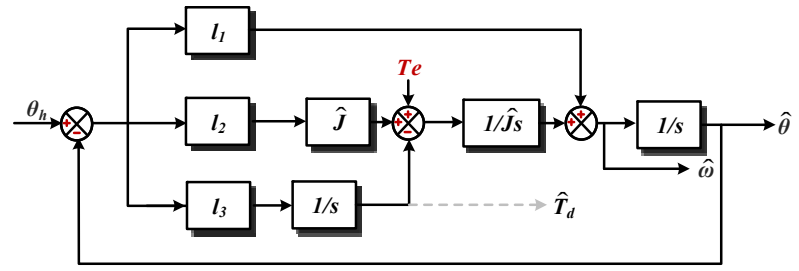

Fig. 3. Block diagram of Luenberger position observer

$$
\begin{aligned}
& \theta_{h}=\frac{1-e^{-s \Delta t}}{s} \theta, \\
& T_{e}=J_{s} \omega .
\end{aligned}
$$

From (9), the transfer function for rotor speed can be deduced as

$$
\hat{\omega}=\frac{J s^{3}+\left(\hat{J} l_{1} s^{2}+\hat{J} l_{2} s-l_{3}\right) \frac{1-e^{-s \Delta t}}{s}}{\hat{J}^{3}+\hat{J} l_{1} s^{2}+\hat{J} l_{2} s-l_{3}} \omega
$$

The error of estimation $\omega$ can be expressed as

$$
\Delta \omega=\omega-\hat{\omega}=\frac{\Delta s^{3}+\left(\hat{J} l_{1} s^{2}+\hat{J}_{2} s-l_{3}\right)\left(1-\frac{1-e^{-s \Delta t}}{s}\right)}{\hat{J}^{3}+\hat{J} l_{1} s^{2}+J l_{2} s-l_{3}} \omega
$$

where, $\Delta J=\hat{J}-J$.

From (11), it can be seen that the estimated rotor speed error is affected by both $\Delta t$ and $\Delta J$. In servo systems, however, the moment of inertia varies slightly in a specific application, and it can be estimated by off-line method, so it can be assumed known. For outputting speed estimation at every control period $T_{s}$, observer input $\theta_{h}$ varies in step manner, it can be regarded as a piecewise constant signal during every Hall interval $\Delta t$. And $\Delta t$ is much bigger than $T_{s}$ during zero to low speed range. Therefore, $\Delta t$ must be considered, and this is the reason for the existence of bumps. Experimental results of bumps in the speed estimation related to $\Delta t$ will be illustrated in the following Section.

\section{Proposed SCHEME}

To enhance the performance of the rotor position estimation, an improved optimal curve fitting approach based on least square method is developed. Also, a real-time reconstruction strategy is adopted to determine the degree of the polynomials automatically at different motion conditions, and a gradual adjustment method is proposed as the updating strategy to eliminate the angle step if a new polynomial is determined based on the last approximate value.

\section{A. Curve fitting based position estimator}

From the Hall sensors data, an appropriate polynomial approximation to the position/time data relationship can be deduced, and this approximation is then used to generate a continuous estimation of rotor position.

There are six states of Hall signals in one electrical period, so a polynomial for fitting seven discrete time/position data $\left(\left\{\left(t_{k-6+i}, \quad \theta_{h}(k-6+i)\right\}_{i=0,1,2, \cdots, 6}\right) \quad\right.$ is considered[17]. The $n$-degree polynomial can be regarded as an approximation signal of the actual angular output in the time-limited interval $\left[t_{k-6}, t_{k}\right]$, such that $n<7$. The polynomial of degree $n$ can be written as 


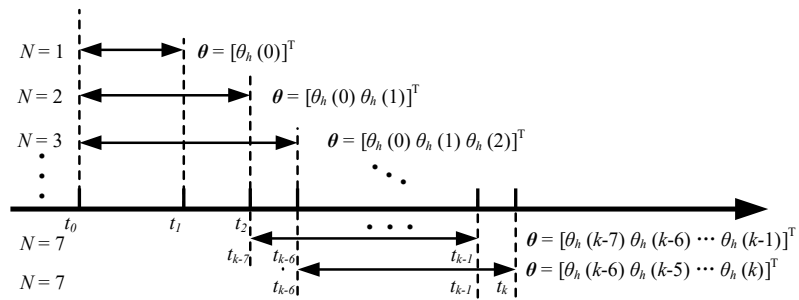

a)

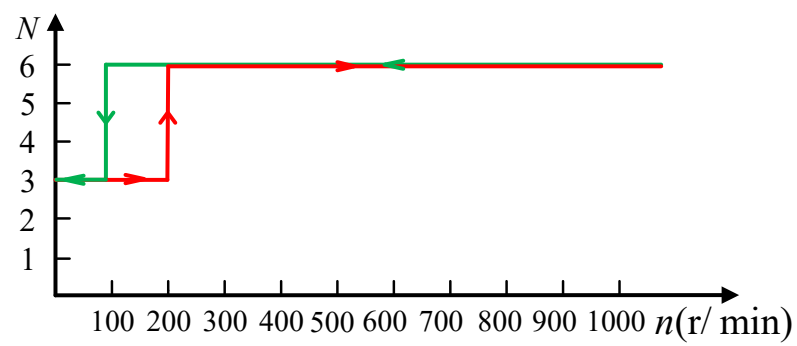

b)

Fig. 4. Real-time reconstruction strategy: a) moving window update manner b) the length setting of the fitting window

$$
y_{k}(t)=a_{0}+a_{1} t+a_{2} t^{2}+\cdots+a_{n} t^{n}
$$

In positioning system, as well as some household applications, the velocity loop is controlled in trapezoid or step manner. Also, the variation of load torque can be ignored when the load torque varies much more slowly compared to the variation of other state variables and the coefficient friction can be neglected in most speed regions. Here, when the motor and load torques are equal, the rotor acceleration is zero and the drive runs at constant speed. However, if the motor and load torque are unequal, the drive accelerates or decelerates. Therefore, by considering the aforementioned assumption, the degree of the fitting curve is restricted to cube $(n \leq 3)$ in this work as a compromise between accuracy and efficiency.

At time instant $t_{k}, \theta_{h}(k)$ is sampled from Hall sensors, and the new polynomial coefficients in (12) can be obtained by adopting the least square fitting approach. Therefore, the fitting problem can be formulated by:

$$
\underset{a}{\operatorname{minimize}}:\left\|\left[\begin{array}{c}
\theta_{p}\left(t_{k-6}\right)-\theta_{h}(k-6) \\
\vdots \\
\theta_{p}\left(t_{k-3}\right)-\theta_{h}(k-3) \\
\vdots \\
\theta_{p}\left(t_{k}\right)-\theta_{h}(k)
\end{array}\right]\right\|_{2}
$$

with variables $\boldsymbol{a}=\left[\begin{array}{llll}a_{0} & a_{1} & \cdots & a_{n}\end{array}\right]^{T}$.

The problem of (13) can be expressed by:

$$
\underset{a}{\operatorname{minimize}}:\|\boldsymbol{T} \boldsymbol{a}-\boldsymbol{\beta}\|_{2}
$$

where

$$
\boldsymbol{T}=\left[\begin{array}{cccc}
1 & t_{k-6} & t_{k-6}^{2} & t_{k-6}^{3} \\
1 & t_{k-5} & t_{k-5}^{2} & t_{k-5}^{3} \\
\vdots & \vdots & \vdots & \vdots \\
1 & t_{k} & t_{k}^{2} & t_{k}^{3}
\end{array}\right], \boldsymbol{\beta}=\left[\begin{array}{c}
\theta_{h}(k-6) \\
\theta_{h}(k-5) \\
\vdots \\
\theta_{h}(k)
\end{array}\right]
$$

The polynomial function is evaluated continuously to give an extrapolated position estimation over the time interval

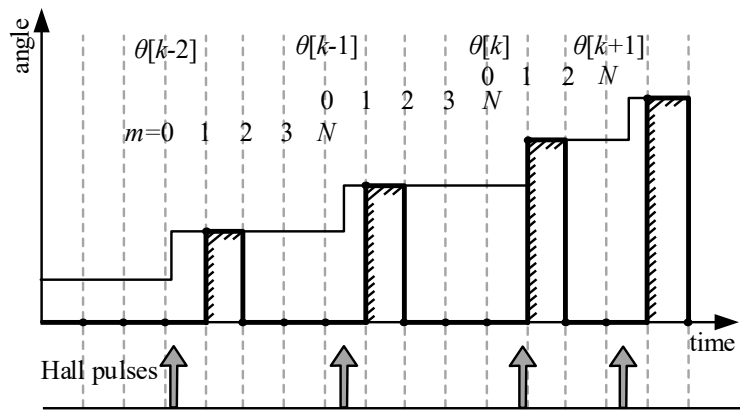

Fig. 5. Timing chart of the output from Hall sensors

beginning at $t_{k}$ and ending when the next Hall signal arrives, say, from $t_{k}$ to $t_{k+1}$. For forward motion of the rotor, the next Hall signal is usually produced at the position $\left(\theta_{h}(k)+60^{\circ}\right)$.

To realize a smooth and steady performance at low speed region and at the start-up process, the length $(\mathrm{N})$ of the fitting window should be speed varied. This is due to the fact that in practice the total torque may varies nonlinearity during a long time interval at low speed region. Fig.4 shows the real-time reconstruction strategy. As shown above, $\theta_{p}(t)$ is determined from the data $\boldsymbol{\theta}$, where $N$ is varied based on the time interval of last Hall sector. By considering variations of the fitting window's length and the motion character in low speed region, fitting problem can be reformulated by:

$$
\underset{a}{\operatorname{minimize}}:\|\boldsymbol{T a}-\boldsymbol{\beta}\|_{2}^{2}+\eta\|\boldsymbol{a}\|_{1}
$$

where $\eta$ is the weight factor, the following $\ell_{1}$-norm regularization method is exploited to reduce the number of nonzero coefficients.

\section{B. Multi-sampling-rate observer based velocity estimator}

In real time implementation, the timing chart of the output of Hall sensors is shown in Fig. 5. The variables $T_{s}$, $T_{1}$, and $T_{w}$ denote the control periods, the interval between two consecutive pulses read by the DSP and the actual interval between two consecutive Hall pulses. The sampling instant $[k, m]$ is defined by

$$
t=\sum_{a=0}^{k} T_{1}\left(\theta\left(t_{a}\right)\right)+m T_{s}=[k, m]
$$

where $k$ and $m$ denote the index number of the Hall signal, and the sampling instant the counting of which starts when a signal edge is detected and reset when the next pulse is detected, respectively. The value of $\mathrm{n}$ is between 0 and $N$, where $N$ denotes last sampling instant. The relationship between the sampling indices $k$ and $m$ can be concluded as

$$
[k, N]=[k+1,0]
$$

During whole speed region, DSP may not read a new position signal in each sampling, and $T_{1}>T_{s}$. When DSP does not read a new Hall pulse, the old position read by DSP in this sampling has an error compared with the actual position, which means that the position read by DSP is unreliable. Then, the measurement value should be ignored. Since accurate position is available only at the moment of Hall pulse input, it is nature to transform (7) in discrete form with sampling period $T_{1}$ as 


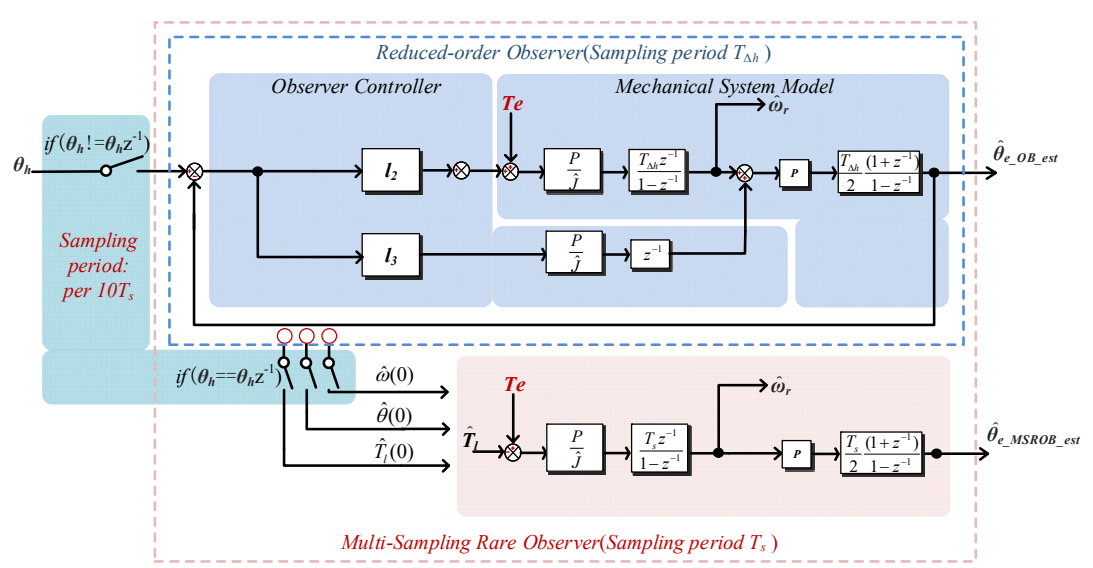

a)

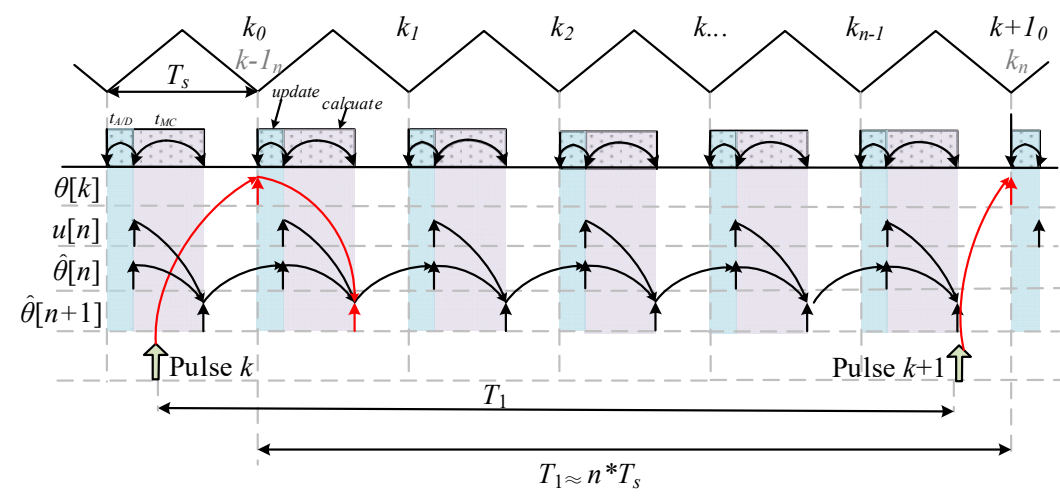

b)

Fig. 6. a) block diagram of reduced-order multi-sampling rate observer b) execution sequence chart of proposed observer

$$
\begin{aligned}
{\left[\begin{array}{l}
\mathbf{x}_{1}(k+1) \\
\mathbf{x}_{2}(k+1)
\end{array}\right] } & =\left[\begin{array}{ll}
\mathbf{A}_{11}^{1} & \mathbf{A}_{12}^{1} \\
\mathbf{A}_{21}^{1} & \mathbf{A}_{22}^{1}
\end{array}\right]\left[\begin{array}{l}
\mathbf{x}_{1}(k) \\
\mathbf{x}_{2}(k)
\end{array}\right]+\left[\begin{array}{l}
\mathbf{B}_{1}^{1} \\
\mathbf{B}_{2}^{1}
\end{array}\right] \mathbf{u} \\
\mathbf{A}^{1} & =\left[\begin{array}{l|l}
\mathbf{A}_{11}^{1} & \mathbf{A}_{12}^{1} \\
\hline \mathbf{A}_{21}^{1} & \mathbf{A}_{22}^{1}
\end{array}\right]=\left[\begin{array}{c|cc}
1 & T_{1} & T_{1}^{2} / 2 J \\
\hline 0 & 1 & T_{1} / J \\
0 & 0 & 1
\end{array}\right] \\
\mathbf{B}^{1} & =\left[\begin{array}{c}
\mathbf{B}_{1}^{1} \\
\mathbf{B}_{2}^{1}
\end{array}\right]=\left[\begin{array}{c}
T_{1}^{2} / 2 J \\
T_{1} / J \\
0
\end{array}\right]
\end{aligned}
$$

Where $\mathbf{x}=\left[\begin{array}{lll}\theta & \omega & T_{\mathrm{L}}\end{array}\right]^{\mathrm{T}}$ is the state variant; $\mathbf{u}=\left[T_{e}\right]$ is the motor torque signal. The observability matrix $\mathbf{O}(\mathbf{C}, \mathbf{A})$ is full rank $\left(\operatorname{det}(\mathbf{O})=T_{1}^{3} / J\right)$, so that it is possible to construct a full state variable estimator, given by

$$
\hat{\mathbf{x}}[k+1]=\mathbf{A}^{1} \hat{\mathbf{x}}[k]+\mathbf{B}^{1} \mathbf{u}[k]+\mathbf{L}^{1}(\mathbf{y}[k]-\hat{\mathbf{y}}[k])
$$

where $\mathbf{y}[k]$ denotes the real position from the Hall sensors, $\hat{\mathbf{y}}[k]=\mathbf{C} \hat{\mathbf{x}}[k]$ denotes the estimated position from the

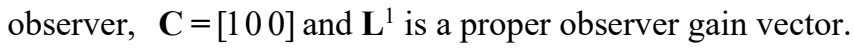

A discrete observer must use a sufficiently long sampling period to match the availability of Hall sensors information, which will lead a poor speed control performance. To address this long sampling problem, it is desirable to estimate velocity information at every sample period $T_{s}$ for the satisfaction of control requirement. As a result, the multi-sampling-rate observer is adopted to predict and correct the errors at every $T_{s}$ sampling instant. In other words, the angular position read by DSP is used to correct the estimation errors at each $T_{1}$ instant, and system model is used as a predictor for state variables, i.e. fig.6. b), as shown in $(21)$

$$
\hat{\mathbf{x}}[m+1]=\left\{\begin{array}{l}
\mathbf{A}^{s} \hat{\mathbf{x}}[m]+\mathbf{B}^{s} \mathbf{u}[m]+\mathbf{L}^{s}\left(\mathbf{y}[m]-\mathbf{C}^{s} \hat{\mathbf{x}}[m]\right), \quad m=0 \\
\mathbf{A}^{s} \hat{\mathbf{x}}[m]+\mathbf{B}^{s} \mathbf{u}[m], \quad m=1,2, \ldots, N-1
\end{array}\right.
$$

where $\mathbf{A}^{\mathrm{s}}, \mathbf{B}^{\mathrm{s}}, \mathbf{C}^{\mathrm{s}}$ and $\mathbf{L}^{\mathrm{s}}=\left[\begin{array}{lll}l_{1} & l_{2} & l_{3}\end{array}\right]^{\mathrm{T}}$ denote the matrices in discrete-time domain sampled by $T_{\mathrm{s}}$.

A full order observer reconstructs all the state variables. However, in practice, the angular position is accurately measured and need not to be estimated at each $T_{1}$ instant when error correction works. Therefore, in the case of detecting angular velocity, a reduced-order observer is suitable. From (19), a reduced-order estimator becomes

$$
\hat{\mathbf{x}}_{2}[m+1]=\mathbf{A}_{22}^{s} \hat{\mathbf{x}}_{2}[m]+\mathbf{B}_{2}^{s} \mathbf{u}[m]
$$

With proper feedback components and gain vector $\mathbf{K}^{\mathrm{s}}$, observer can be designed as follows:

$$
\hat{\mathbf{x}}_{2}[m+1]=\mathbf{A}_{22}^{s} \hat{\mathbf{x}}_{2}[m]+\mathbf{B}_{2}^{s} \mathbf{u}[m]+\mathbf{K}^{s}\left(\mathbf{A}_{12}^{s} \mathbf{x}_{2}[m]-\mathbf{A}_{12}^{s} \hat{\mathbf{x}}_{2}[m]\right)
$$

For error correction only works at time instant $m=0$, the actual state variables $\mathbf{x}_{2}$ are unknown, so replace $\mathbf{A}_{12} \mathbf{x}_{2}$ [0] by accessible term $\mathbf{x}_{1}[0]$ from equation (19a), so defining position error $\Delta \mathbf{x}_{1}[0]=\mathbf{x}_{1}[0]-\hat{\mathbf{x}}_{1}[0],(19 a)$ can be written as

$$
\mathbf{A}_{12} \mathbf{x}_{2}[0]=\mathbf{x}_{1}[1]-\mathbf{x}_{1}[0]-\mathbf{B}_{1} \mathbf{u}[0]
$$




$$
\mathbf{A}_{12} \hat{\mathbf{x}}_{2}[0]=\hat{\mathbf{x}}_{1}[1]-\left(\Delta \mathbf{x}_{1}[0]+\hat{\mathbf{x}}_{1}[0]+\mathbf{B}_{1} \mathbf{u}[0]\right)
$$

Finally, with (24) and (25), (23) can be performed as

$$
\hat{\mathbf{x}}_{2}[1]=\mathbf{A}_{22} \hat{\mathbf{x}}_{2}[0]+\mathbf{B}_{2} \mathbf{u}[0]+\mathbf{L}\left(\mathbf{x}_{1}[1]-\hat{\mathbf{x}}_{1}[1]\right)
$$

According to equation form (23) to (26), we can conclude the proposed speed observer as expressed in equation (27), and the block diagram of the observer is shown in Fig.6. a).

The frequency of the Hall signal transitions between the six quantized states is speed-dependent, so $T_{1}$ is variable. As a result, the observer gain should be determined by a tradeoff between estimation performance and estimation noise response. On one hand, the bandwidth of the observer should not be too high, causing the estimated velocity to track the quantized nature of the input and be erroneous. On the other hand, relatively high bandwidth is usually desired in order to obtain good dynamic estimation of disturbances. Thus, to maximize performance while limiting errors, pole assignment can be achieved by a consideration of the error dynamics of the observer.

Rearranging (27) based on the sampling instant $m=N-1$, the state variable can be expressed as

$$
\begin{aligned}
\hat{\mathbf{x}}_{2}[N] & =\mathbf{A}_{22}^{s{ }^{N}} \hat{\mathbf{x}}_{2}[0]+\mathbf{A}_{22}^{s N-1} \mathbf{B}_{2}^{s} \mathbf{u}[0]+\mathbf{A}_{22}^{s N-2} \mathbf{B}_{2}^{s} \mathbf{u}[1]+\cdots \\
& +\mathbf{A}_{22}^{s}{ }_{20}^{0} \mathbf{B}_{2}^{s} \mathbf{u}[N-1]+\mathbf{A}_{22}^{s N-1} \mathbf{K}^{s}\left(\mathbf{A}_{12}^{s} \mathbf{x}_{2}[0]-\mathbf{A}_{12}^{s} \hat{\mathbf{x}}_{2}[0]\right)
\end{aligned}
$$

The state vector of plant can be written with the same form in discrete time domain as

$$
\begin{aligned}
\mathbf{x}_{2}[N] & =\mathbf{A}_{22}^{s N} \mathbf{x}_{2}[0]+\mathbf{A}_{22}^{s N-1} \mathbf{B}_{2}^{s} \mathbf{u}[0]+\mathbf{A}_{22}^{s N-2} \mathbf{B}_{2}^{s} \mathbf{u}[1] \\
& +\cdots+\mathbf{A}_{22}^{s 0} \mathbf{B}_{2}^{s} \mathbf{u}[N-1]
\end{aligned}
$$

Subtracting (29) from (28), the error of estimation $\mathbf{e}$ of a sampling frame $T_{1}$ can be expressed as

$$
\mathbf{e}[k+1]=\left(\mathbf{A}_{22}^{s N}-\mathbf{A}_{22}^{s N-1} \mathbf{K}^{s} \mathbf{A}_{12}^{s}\right) \mathbf{e}[k]
$$

Consequently, the observer gains are obtained by placing the poles in the unit circle and solving the equation

$$
\prod_{i=1}^{q}\left(z-z_{i}\right)=\left|z \mathbf{I}-\mathbf{A}_{22}^{s N-1}\left(\mathbf{A}_{22}^{s}-\mathbf{K}^{s} \mathbf{A}_{12}^{s}\right)\right|
$$

Where $z_{i}$ and $q$ denotes the $i$ th pole on $z$-plane and number of poles, respectively.

\section{EXPERIMENT RESULTS}

The experiment platform is composed of a $750 \mathrm{~W}$ motor, whose parameters are given in Table I, IGBT full bridge inverter and 32 bit floating point DSP TMS320F28335. The inverter is powered by single phase $220 \mathrm{v}$, and the switching frequency is $10 \mathrm{KHz}$. In order to verify the accuracy of estimation result, the low cost hall position sensor and an encoder with 10000 pulses per revolution. As shown in Fig.7
TABLE I. SPECIFICATION OF SPM

\begin{tabular}{|c|c|}
\hline Quantity & Value $[$ Unit $]$ \\
\hline Pole & 8 \\
\hline Rate torque & $2.39[\mathrm{~N} \mathrm{~m}]$ \\
\hline Base speed & $3000[\mathrm{r} / \mathrm{min}]$ \\
\hline Rated current & $3[\mathrm{~A}]$ \\
\hline Voltage constant & $48[\mathrm{~V} / 1000 \mathrm{r} / \mathrm{min}]$ \\
\hline Stator resistance & $2.88[\Omega]$ \\
\hline Stator inductance & $6.4[\mathrm{mH}]$ \\
\hline Rotational inertia & $0.001638\left[\mathrm{Kg} \cdot \mathrm{m}^{2}\right]$ \\
\hline
\end{tabular}

the Hall signals distribute averagely during one electrical period in the target machine.

\section{A. Position estimator performance}

The position estimation performance by average speed method and proposed curve fitting based method are demonstrated in Fig.8 (a) and (b), respectively. Severe phase current distortion can be observed in Fig.8 (a), which is caused by inaccurate position estimation. In average speed method, the real position information is updated at the edges of hall signals. At this point the estimated position largely mismatches with the real one, leading to the undesired current distortion. While by adopting proposed curve fitting method, the position estimation accuracy is greatly improved since more data is used in the estimation process as well as increase the polynomial order. This is the reason why much better performance is obtained in Fig.8 (b).

The real position, estimated position using proposed curve fitting method, and position error in the speed reversal process are demonstrated in Fig.9. It gives that in steady state, the position estimation error is very small within the range of only 0.066rad (around 3.8 degree). Even if in the transient state, the position error is also acceptable, with the largest error is only $0.182 \mathrm{rad}$ (around 10.4 degree). This result further confirms the effectiveness and accuracy of proposed curve fitting based method.

\section{B. Speed estimator performance}

The speed estimation performances by Luenberger observer (LOB) and proposed multi-sampling-rate observer (MSROB) are demonstrated in Fig.10. The red waveforms represent the estimated speed coming from LOB, while the blue curves represent the estimated speed of MSROB. The estimated speed of LOB has obvious oscillation, which is caused by the discontinuity of LOB input. In addition, it is worth to note that the speed difference between maximum and minimum value of LOB decrease from $30.864 \mathrm{rpm}$ to 6.4 $\mathrm{rpm}$ as the speed incresae from $300 \mathrm{rpm}$ to $3000 \mathrm{rpm}$. This can be explained that as the increase of speed, the sampling rate of Hall sensor will incresae as well.

$$
\begin{aligned}
& \hat{\mathbf{x}}_{1}[m+1]= \begin{cases}\mathbf{x}_{1}[m+1], & m=0 \\
\hat{\mathbf{x}}_{1}[m]+\mathbf{A}_{12}^{s} \mathbf{x}_{2}[m]+\mathbf{B}_{1}^{s} \mathbf{u}[m], & m=1,2, \ldots, N-1\end{cases} \\
& \hat{\mathbf{x}}_{2}[m+1]= \begin{cases}\mathbf{A}_{22}^{s} \hat{\mathbf{x}}_{2}[m]+\mathbf{B}_{2}^{s} \mathbf{u}[m]+\mathbf{K}^{s}\left\{\mathbf{x}_{1}[m+1]-\left(\hat{\mathbf{x}}_{1}[m]+\mathbf{A}_{12}^{s} \mathbf{x}_{2}[m]+\mathbf{B}_{1}^{s} \mathbf{u}[m]\right)\right\}, & m=0 \\
\mathbf{A}_{22}^{s} \hat{\mathbf{x}}_{2}[m]+\mathbf{B}_{2}^{s} \mathbf{u}[m], & m=1,2, \ldots, N-1\end{cases}
\end{aligned}
$$




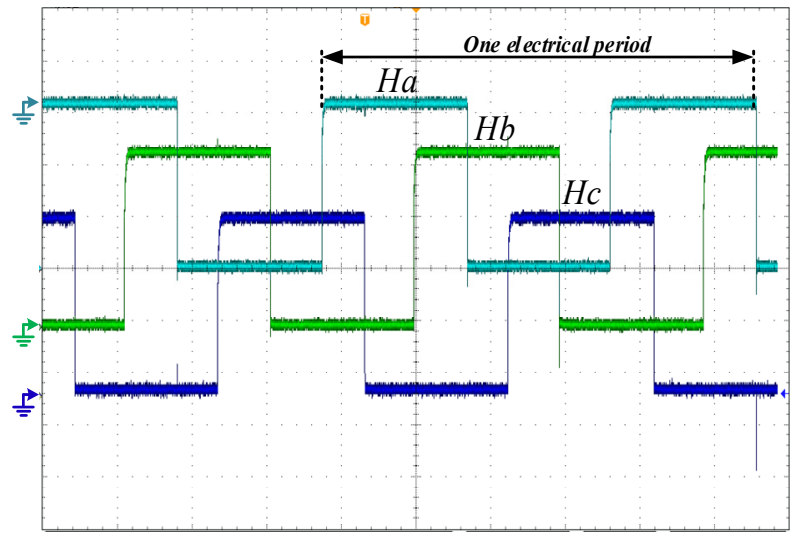

Fig. 7. The discrete output of 3 binary Hall sensors

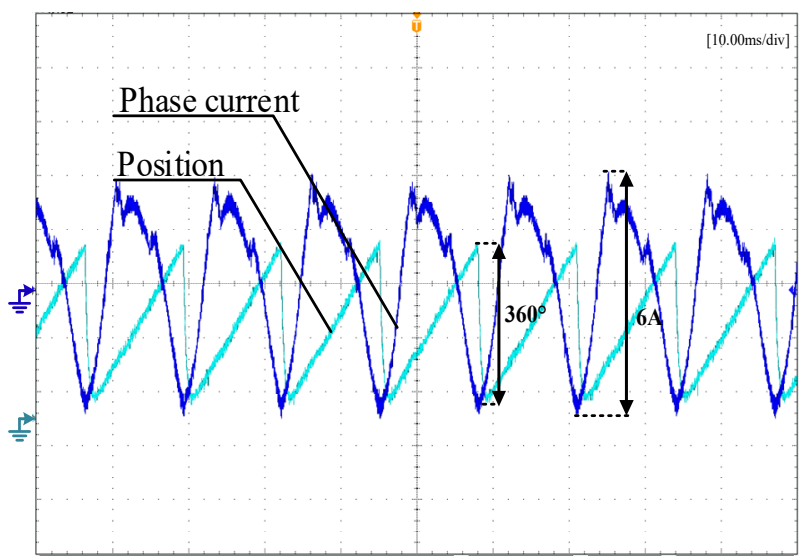

a)

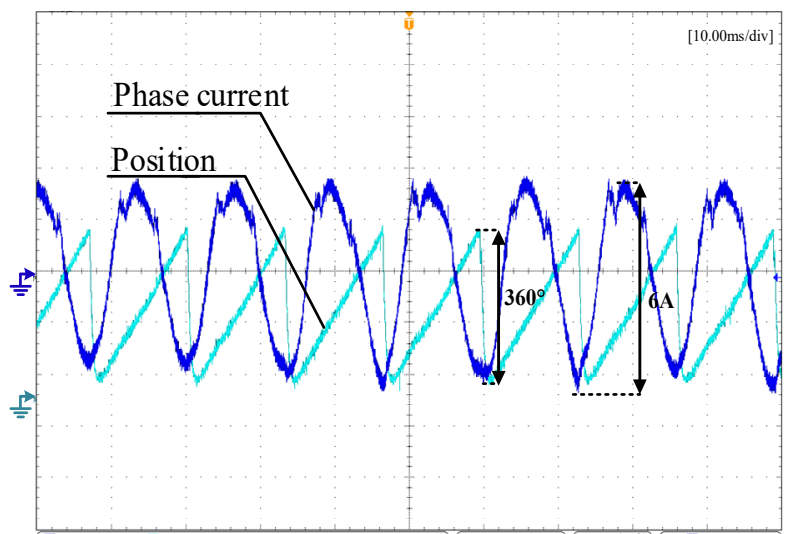

b)

Fig. 8. Phase current waveform and rotor position at constant speed: a) with average speed method. b) with the proposed method.

However, by adopting the proposed MSROB, the influence of discontinuous effect of input will be weakened due to the multi-sampling character of proposed observerexplains why the estimated speed of MSROB is much smoother compared with that of LOB.

\section{Servo operation}

To verify the feasibility of proposed idea in the servo application, the position control experiment is carried out as shown in Fig.11. The position reference is given in a step manner. The actual position which is obtained from a 10000 pluses/rev encoder, the estimated position from proposed method, and the $q$-axis currents are exhibited. The results confirm that with the proposed idea, the low resolution Hall sensor can be successfully used in servo application.

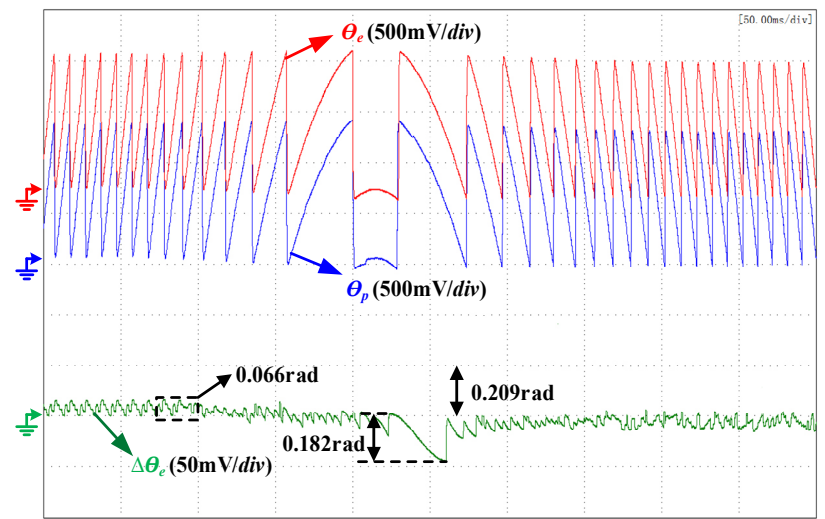

Fig.9. Position estimation performance at speed reversal process with proposed method

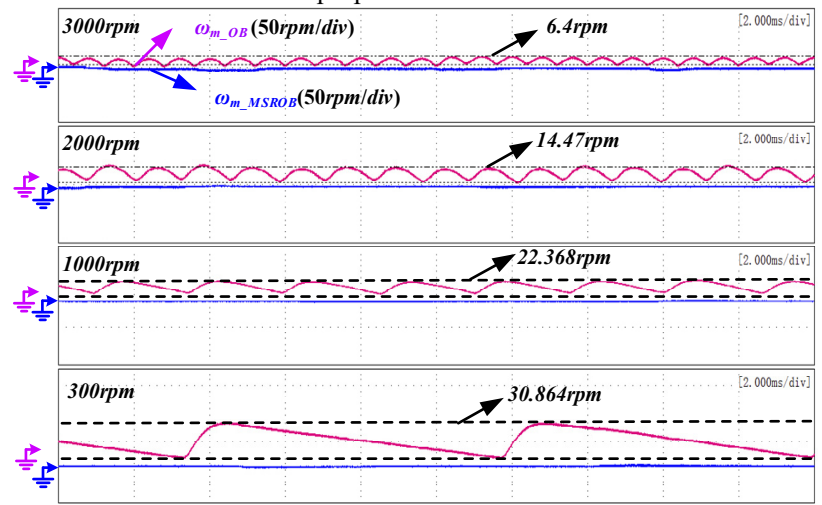

Fig.10. The discrete output of 3 binary Hall sensors

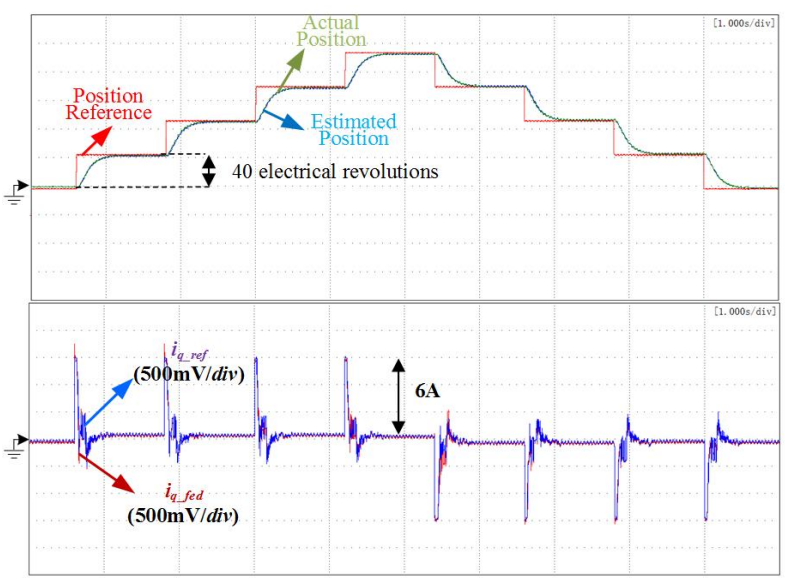

Fig. 11. Position loop control performance with proposed approach

\section{CONCLUTION}

In this paper, the drawbacks of conventional model-free and model-based position/speed estimation methods in practical applications are analyzed. Based on this, a new method that combines them together is proposed, where model-free method is for position estimation and modelbased method is for speed estimation. Besides, some compensation ideas are carried out to confirm a favorable close loop control effect within a wide speed range, which can satisfy the needs for majority low cost servo, electric vehicle and household appliance. The feasibility of the proposed method is verified by experimental verifications. Experimental results show that compared with the conventional methods, the proposed idea is able to smoothly manage the speed and position estimation. In addition, the closed-loop servo operation performance is also desirable. 
Experimental results highlight this promising method in the low cost servo applications.

\section{REFERENCES}

[1] Murakami, Sohji, et al. "Encoderless servo drive with adequately designed IPMSM for pulse voltage injection based position detection." Energy Conversion Congress and Exposition (ECCE), 2011 IEEE. IEEE, 2011.

[2] Mercorelli P. A two-stage sliding-mode high-gain observer to reduce uncertainties and disturbances effects for sensorless control in automotive applications[J]. IEEE Transactions on Industrial Electronics, 2015, 62(9): 5929-5940.

[3] Wang G, Xiao D, Zhao N, et al. Low-Frequency Pulse Voltage Injection Scheme-Based Sensorless Control of IPMSM Drives for Audible Noise Reduction[J]. IEEE Transactions on Industrial Electronics, 2017, 64(11): 8415-8426.

[4] Makino S, Kakihara M, Takase Y, et al. Development of a StatorMagnetless Linear Synchronous Motor for Sensorless Control[J]. IEEE Transactions on Industry Applications, 2017, 53(5): 4559-4568.

[5] Xu D, Wang B, Zhang G, et al. A review of sensorless control methods for AC motor drives[J]. CES Transactions on Electrical Machines and Systems, 2018, 2(1): 104-115.

[6] De Donato G, Scelba G, Pulvirenti M, et al. Low-Cost, HighResolution, Fault-Robust Position and Speed Estimation for PMSM Drives Operating in Safety-Critical Systems[J]. IEEE Transactions on Power Electronics, 2018.

[7] H. J. Ahn and D. M. Lee, "A New Bumpless Rotor-Flux Position Estimation Scheme for Vector-Controlled Washing Machine," in IEEE Transactions on Industrial Informatics, vol. 12, no. 2, pp. 466473, April 2016.

[8] S. Y. Kim, C. Choi, K. Lee and W. Lee, "An Improved Rotor Position Estimation With Vector-Tracking Observer in PMSM Drives With Low-Resolution Hall-Effect Sensors," in IEEE Transactions on Industrial Electronics, vol. 58, no. 9, pp. 4078-4086, Sept. 2011.
[9] R. Raja, T. Sebastian, M. Wang, A. Gebregergis and M. S. Islam, "Effect of Position Sensor Error on the Performance of Permanent Magnet Machine Drives," in IEEE Transactions on Industry Applications, vol. 53, no. 6, pp. 5518-5526, Nov.-Dec. 2017.

[10] D. Reigosa, D. Fernandez, C. Gonzalez, S. B. Lee and F. Briz, "Permanent magnet synchronous machine drive control using analog hall-effect sensors," 2017 IEEE Energy Conversion Congress and Exposition (ECCE), Cincinnati, OH, 2017, pp. 3966-3971.

[11] D. M. Lee, D. C. Lim and H. J. Ahn, "Position linearisation scheme for permanent magnet synchronous motor drive of washing machine using low-resolution hall sensors," in Electronics Letters, vol. 51, no. 22, pp. 1765-1767, 10222015.

[12]

[13] Dalala, Zakariya M., Younghoon Cho, and Jih-Sheng Lai. "Enhanced vector tracking observer for rotor position estimation for PMSM drives with low resolution Hall-Effect position sensors." Electric Machines \& Drives Conference (IEMDC), 2013 IEEE International. IEEE, 2013.

[14] Shi T, Wang Z, Xia C. Speed measurement error suppression for PMSM control system using self-adaption Kalman observer[J]. IEEE Transactions on Industrial Electronics, 2015, 62(5): 2753-2763.

[15] Lee D M. Position Estimator Employing Kalman Filter for PM Motors Driven with Binary-type Hall Sensors[J]. Journal of Electrical Engineering \& Technology, 2016, 11(4): 931-938.

[16] Zhu H, Sugie T. Velocity estimation of motion systems based on lowresolution encoders[J]. Journal of Dynamic Systems, Measurement, and Control, 2013, 135(1): 011006.

[17] Feng, Zhaodong, and Paul P. Acarnley. "Extrapolation technique for improving the effective resolution of position encoders in permanentmagnet motor drives." IEEE/ASME Transactions on Mechatronics 13.4 (2008): 410-415.

[18] Zhang, Xinghao, and Wei Zhang. "An improved rotor position estimation in PMSM with low-resolution hall-effect sensors." Electrical Machines and Systems (ICEMS), 2014 17th International Conference on. IEEE, 2014. 